\title{
The Psychosocial Complications of Vitiligo
}

\section{David J Chandler ${ }^{1 *}$, Reena Shah ${ }^{2}$ and Anthony Bewley ${ }^{3}$}

${ }^{1}$ Clinical Research Fellow (Dermatology), Weatherall Institute of Molecular Medicine, University of Oxford, Oxford, OX3 9DS, UK

${ }^{2}$ Chartered Clinical Psychologist, Royal London Hospital, Whitechapel, London, E1 1BB, UK

${ }^{3}$ Consultant Dermatologist, Royal London Hospital, Whitechapel, London, E1 1BB, UK

Vitiligo is an acquired chronic depigmentation disorder characterised by white skin patches. The prevalence is around $1 \%$ in Europe and the United States, and more than $8 \%$ in parts of India. Vitiligo usually occurs after birth and the average age of onset is around 20 years. Vitiligo commonly affects the face and extremities and is often immediately visible to others hence evokes high levels distress associated with appearance concern. Both sexes are affected equally, although women are more likely to seek treatment [1]. Disease progression is unpredictable and response to treatment is highly variable.

The psychosocial impact of vitiligo is comparable to that of other common skin diseases such as psoriasis and eczema. Within the clinical guidelines, the main impact of vitiligo is the psychological effect of the disease [2]. For example, a review by Ongenae et al. indicated that high levels of emotional responses were reported, such as increased selfconsciousness, lower self-esteem, higher levels of perceived stigma and disability, anger, poorer Quality Of Life (QOL) overall and negative impact on sexual relationships [3]. Within a UK survey the majority of patients reported that vitiligo moderately or severely affected their QOL [4]. Patients often report feeling distressed and embarrassed about their appearance, which can lead to low self-esteem, fear of rejection and social withdrawal. The key factors in this process are how people living with vitiligo interpret it, how they interpret themselves and how they interpret and respond to the experience of being in a social situation. In particular, patients have difficulties with social anxiety and high level of appearance related concern [5]. These difficulties can cause a person with vitiligo to experience a range of negative emotions and demonstrate certain behaviours and thoughts that can perpetuate psychological distress. A study by Schmid-Ott et al. indicated that patients with vitiligo used avoidant coping styles compared to controls [6]. Some strategies appear to be more psychologically helpful than others, as avoidance and concealment can exacerbate distress. Concealment through the use of cosmetic creams or selective choice of clothing may enable people to engage in activities that they may have avoided. However these strategies have short-term positive consequences and the underlying issues remain. The psychosocial consequences are often more significant for females, with detrimental effects particularly on sexual relationships and perceived suitability for marriage. Characteristics commonly associated with a lower QOL in patients with vitiligo include darker skin colour (Fitzpatrick skin type IV-VI), greater skin involvement, more visible lesions, longer disease duration and previous failed treatments [1]. Some studies have suggested that the impact of vitiligo on psychosocial well-being is greater in early childhood during the formative years; others argue that early age of onset is a protective factor as it allows for the development of coping mechanisms at a time when physical appearance is less of a concern [7]. A study conducted in India showed that patients with vitiligo had a range of concerns including how the disease would progress and its implications on social participation, employment and marriage opportunities [8]. There were several misconceptions about the cause of vitiligo however most patients did not think the disease was contagious or related to leprosy. Misconceptions and negative attitudes around vitiligo can cause increased emotional distress and heightened feelings of rejection and isolation for those affected.

Vitiligo is associated with high psychiatric comorbidity. Studies conducted in India have shown that 1 in 4 patients with vitiligo have psychiatric morbidity $[9,10]$. Major depression, anxiety and adjustment disorder are among the most common conditions experienced by patients. Suicidal ideation has been reported. A survey administered to members of The Vitiligo Society (a UK patient support group) revealed that $20 \%$ of patients were depressed about their vitiligo.

Although finding a cure or effective treatment is a high priority for most patients, 4,8 patients often experience indifference from the doctors toward their skin problem and do not feel adequately supported by them [2]. The assessment of patients with vitiligo should take into consideration the wide range of psychosocial comorbidities that can occur. This can promote normalisation of the impact of living with the condition, can possibly educate the patient about the connection between the mind and the skin and offer the patient a safe place to be listened to.

Assessment of disease severity by doctor and patient are often inconsistent, which probably reflects the significant contribution of psychological factors to overall morbidity. Perceived disease severity and personality are known predictors of QOL impairment in patients with vitiligo. The development in recent years of vitiligo-specific tools for assessing QOL (VitiQoL) and psychological burden (vitiligo impact scale) is encouraging $[11,12]$. VitiQoL scores correlate strongly with self-reported disease severity. Psychosocial and QOL assessment is essential and patients should be regularly screened for symptoms of depression and anxiety, for example, to facilitate early treatment and to ensure that both medical and psychological needs of the patient are addressed. Treatment of psychosocial comorbidities is also important and patients should preferably receive integrated management from dermatologists and mental health professionals. Ideally, having dedicated psychologists in dermatology would help reduce the stigma and negative perceptions of mental health and promote the importance of integrated holistic treatment. Guidelines for the management of vitiligo recommend psychological interventions, specifically cognitive behavioural therapy (CBT) [13]. Self-help based on social anxiety associated with living with vitiligo has also been shown to be effective, however further research is required [5]. Counselling/psychological services should be offered to patients and where appropriate to the parents of affected children.

*Corresponding author: David J Chandler, Clinical Research Fellow (Dermatology) Weatherall Institute of Molecular Medicine, University of Oxford, Oxford, United Kingdom, Tel: +447788698976; E-mail: david@queenspoint.com

Received August 22, 2014; Accepted September 11, 2014; Published September 13,2014

Citation: Chandler DJ, Shah R, Bewley A (2014) The Psychosocial Complications of Vitiligo. Pigmentary Disorders 1:130. doi:10.4172/JPD.1000130

Copyright: (c) 2014 Chandler DJ. This is an open-access article distributed under the terms of the Creative Commons Attribution License, which permits unrestricted use, distribution, and reproduction in any medium, provided the original author and source are credited. 


\section{References}

1. Alikhan A, Felsten LM, Daly M, Petronic-Rosic V (2011) Vitiligo: a comprehensive overview Part I. Introduction, epidemiology, quality of life, diagnosis, differential diagnosis, associations, histopathology, etiology, and work-up. J Am Acad Dermatol 65: 473-491.

2. Gawkrodger DJ, Ormerod AD, Shaw L, Mauri-Sole I, Whitton ME, et al. (2010) Vitiligo: concise evidence based guidelines on diagnosis and management. Postgrad Med J 86: 466-471.

3. Ongenae K, Van Geel N, De Schepper S, Naeyaert JM (2005) Effect of vitiligo on self-reported health-related quality of life. Br J Dermatol 152: 1165-1172.

4. Talsania N, Lamb B, Bewley A (2010) Vitiligo is more than skin deep: a survey of members of the Vitiligo Society. Clin Exp Dermatol 35: 736-739.

5. Shah R, Hunt J, Webb TL, Thompson AR (2014) Starting to develop self-help for social anxiety associated with vitiligo: using clinical significance to measure the potential effectiveness of enhanced psychological self-help. Br J Dermatol 171: 332-337.

6. Schmid-Ott G, Künsebeck HW, Jecht E, Shimshoni R, Lazaroff I, et al. (2007) Stigmatization experience, coping and sense of coherence in vitiligo patients. J Eur Acad Dermatol Venereol 21: 456-461.
7. Hill-Beuf A, Porter JD (1984) Children coping with impaired appearance: social and psychologic influences. Gen Hosp Psychiatry 6: 294-301.

8. Pahwa P, Mehta M, Khaitan BK, Sharma VK, Ramam M (2013) The psychosocial impact of vitiligo in Indian patients. Indian J Dermatol Venereol Leprol 79: 679-685.

9. Mattoo SK, Handa S, Kaur I, Gupta N, Malhotra R (2002) Psychiatric morbidity in vitiligo: prevalence and correlates in India. J Eur Acad Dermatol Venereol 16: $573-578$.

10. Mattoo SK, Handa S, Kaur I, Gupta N, Malhotra R (2001) Psychiatric morbidity in vitiligo and psoriasis: a comparative study from India. J Dermatol 28: 424432.

11. Krishna GS, Ramam M, Mehta M, Sreenivas V, Sharma VK, et al. (2013) Vitiligo impact scale: an instrument to assess the psychosocial burden of vitiligo. Indian J Dermatol Venereol Leprol 79: 205-210.

12. Lilly E, Lu PD, Borovicka JH, Victorson D, Kwasny MJ, et al. (2013) Development and validation of a vitiligo-specific quality-of-life instrument (VitiQoL). J Am Acad Dermatol 69: e11-18.

13. Taieb A, Alomar A, Böhm M, Dell'anna ML, De Pase A, et al. (2013) Guidelines for the management of vitiligo: the European Dermatology Forum consensus. Br J Dermatol 168: 5-19. 